\title{
Fleur De Lys Abdominoplasty: Techniques to Reduce Complication Rates - Our Experience
}

TanAlethea, Veeramani Siva and Rao G Sambasiva*

University Hospital North Durham, Durham, United Kingdom

\begin{abstract}
Introduction: Standard abdominoplasties are inadequate in addressing gross tissue excess and laxity. The Fleur De Lys technique addresses supraumbilical excess and is ideal for massive weight loss patients. However, many surgeons hold reservations with this technique because of potentially high risk of complication rates. In our experience, it is a safe technique with low complications with regard to wound healing, with a good patient satisfaction rate.

Aims: This study illustrates an effective modification of the Fleur De Lys technique by a single surgeon in our unit. We aim to share our experiences on the Fleur De Lys abdominoplasty and techniques to reduce complications.

Methodology: We described the senior author's improvised Fleur De Lys operative technique. We obtained medical illustrations outlining pre-operative assessment and markings, and intra-operative techniques. We also discussed selection criteria. We reviewed complications and satisfaction of 29 patients who had undergone Fleur De Lys in our unit over a 3 year period.

Results: Our patient demographic showed as ratio $\mathrm{M}: \mathrm{F}=1: 14$, mean age was 39 years, mean pre-operative BMI $26.8 \mathrm{kgm}^{-2}$ and mean tissue weight excised was $1669 \mathrm{Gm} .17 .2 \%(\mathrm{n}=5)$ had wound breakdown, 6.9\% $(\mathrm{n}=2)$ developed infection and $6.9 \%(n=2)$ had seroma. Only $10.3 \%(n=3)$ patients required revision operation. Our patient satisfaction survey showed 27 out of 29 patients had a positive patient satisfaction response.

Discussion: The Fleur De Lys abdominoplasty is safe in carefully selected patients. The senior author's improvisation 'pinch and reduce by an inch' technique during pre-operative skin marking placement together with a 'no undermining' principle intra-operatively reduces wound edge tension during closure, minimizes risk of skin flap necrosis and reduces potential dead space for fluid collection such as seroma. In our experience, this technique is safe and yields satisfactory results.
\end{abstract}

Keywords: Seroma; Fleur De Lys abdominoplasty

\section{Introduction}

Standard abdominoplasty technique enables excision of excess tissue in one plane only through a single transverse incision. The single incision may be inadequate in addressing excessive tissue [1]. The Fleur De Lys (FDL) technique removes supraumbilical horizontal excess via a vertical excision, in addition to the transverse incision.

The operation derived its name from the first stitch bringing the flaps together, giving the appearance of a fleur-de-lys pattern. A similar operative technique was described as early as 1967 by Castanares and Goethel [2] but the FDL technique was only later popularised by Dellon in 1985 [3]. Dellonused this technique in patients who had marked weight loss, abdominal panniculus with obesity or supra umbilical dermato chalasis [4]. It is therefore an ideal technique for massive weight loss patients [5].

However, in the face of current increasing obesity globally [6], accompanied with an increase in bariatric surgeries, this subset of patient population present different surgical challenges compared to cosmetic driven abdominoplasty. They may have excessive skin in both horizontal and vertical components. In this patient group, a standard abdominoplasty technique may not be the most appropriate surgical approach. The FDL technique is ideal for the massive weight loss patient and should be the surgical approach of choice.

Previous studies have emphasized the high possible complications rates associated with this technique $[1,7,8]$. Supra-epigastric dog ears and wound necrosis at the $\mathrm{T}$ junction are well-known associated complications previously described. Common sense dictates with the addition of excision of a vertical component, this inevitably means an additional vertical wound with potential wound healing problems and an increase in potential dead space, thereby increasing risk of seroma.
Excision of the vertical component also means more perforators are divided during rising of the flap resulting in increased risk of bleeding. For these reasons, many surgeons hesitate to perform the FDL technique. This study describes the senior author's technical tips and modifications on current FDL to reduce these complications, and is a reflection of our experience.

\section{Materials and Methodology}

The senior author provided a description of selection criteria; pre-operative assessment and markings, intra-operative techniques and post-operative management. We reviewed the all patients who had undergone Fleur De Lys abdominoplasty in our unit over a 3 year period, giving us a total of 29 patients. We retrospectively analysed case notes and reviewed clinic letters. We analysed complication rates under the following categories:
1. Early complication rates ( $<2$ weeks)
2. Intermediate complication rates ( $>2$ weeks, $<6$ months)
3. Aesthetic complication rates ( $>6$ months)

*Corresponding author: Rao G Sambasiva, University Hospital North Durham, Durham, United Kingdom, E-mail: Sam.Rao@cddft.nhs.uk

Received March 12, 2013; Accepted April 28, 2013; Published September 28 2013

Citation: Alethea T, Siva V, Sambasiva RG (2013) Fleur De Lys Abdominoplasty: Techniques to Reduce Complication Rates - Our Experience. Surgery Curr Res 3 148. doi:10.4172/2161-1076.1000148

Copyright: $\odot 2013$ Alethea T, et al. This is an open-access article distributed under the terms of the Creative Commons Attribution License, which permits unrestricted use, distribution, and reproduction in any medium, provided the original author and source are credited. 
We compared our experience to several recent published case series of the Fleur De Lys abdominoplasty technique.

\section{Patient selection criteria}

The selection criteria for Fleur De Lys used in our unit are as follows:

1. Massive Weight Loss patients, following either bariatric surgery or, diet and exercise,

2. Presence of excessive skin in supra umbilical region that cannot be adequately corrected by a single lower abdominal transverse incision.

3. Patient's willingness to accept an additional vertical midline scar as well as the transverse scar. They should also be made aware of current evidence based medicine with regards to the complication rates of the Fleur De Lys technique.

4. Presence of divarication of recti does not exclude a patient from a potential Fleur De Lys. The senior author feels that this can be easily corrected intra-operatively.

In addition to careful patient selection, the senior author believes meticulous planning and attention to detail, in addition to his modification of the technique, is key to a minimising complications and maximising successful outcome. The senior author shares his experience of the Fleur De Lys technique as detailed below.

Pre-operative planning and technique: With the patient standing, the lower transverse abdominal skin marking is made, similar to that for the standard abdominoplasty. With the patient in supine position, pinch in the middle of abdomen on either side of the umbilicus. Mark the lateral limits.

Convert this to a vertical ellipse by extending the markings of lateral limits on either side to the xiphisternum superiorly and to the pubic symphisis inferiorly.

As an additional step to the traditional Fleur De Lys markings, the senior author reduces the maximum width of the original vertical ellipse by two inches (reducing an inch on either side, thereby reducing a total of two inches).

Finally, mark the umbilicus. There are various methods of doing this, either the shield sign or oval shaped.

Intra-operative technique: When excising skin and fat, start at the lower border of the lower abdominal transverse incision marking. Raise the abdominal flap, above the fascia, up to the umbilicus and dissect around the umbilicus, taking care to leave it attached to the rectus sheath.

It is usual to come across several vertical perforators coming into the skin during the dissection. Coagulate and or ligate perforators of either side before dividing in the middle. This step is extremely important to reduce complication, in particular bleeding and significant haematoma. If perforators are divided before ligation and/or coagulation, the ends will retract which may cause technical difficulty in achieving adequate haemostasis.

As the vertical skin ellipse excision is narrower than the traditional Fleur De Lys, no undermining of lateral edges is required to close the wound. The senior author does not 'break the table' during closure of the transverse wound. Tension on wound edges during closure increases risks of skin necrosis and dehiscence. Both these steps allow wound closure without tension.

The senior author personally prefers placement of two drains, one in the lower transverse abdominal closure and another under the vertical ellipse excision.

Post-operative care: All our Fleur De Lys patients have an abdominal corset (Tubipad was corset of choice for our unit), placed immediately post operatively. We ensure all patients receive DVT prophylaxis and encourage early mobilisation. Drains are removed when drainage is $<50 \mathrm{mls} / 24$ hours.

\section{Results}

Our patient demographic showed a ratio of $\mathrm{M}: \mathrm{F}=1: 14$. The mean patient age was 39 years, mean pre-operative BMI $26.8 \mathrm{kgm}-2$ and mean tissue weight excised was $1669 \mathrm{Gm} .5$ patients had minor wound breakdown, 2 patients developed infection and 2 developed had seroma. All those who had minor wound breakdown were treated successfully with dressings only. Only $10.3 \%(n=3)$ required revision operation. 27 out of 29 patients achieved satisfactory aesthetic result. No patients developed post-operative haematoma. We found minimal aesthetic complication rates for asymmetry, remaining skin laxity, hypertrophic scarring and dog ears. In particular, no patients had remaining skin laxity and only $3.4 \%(n=1)$ had persistent asymmetry. Only three patients required revision operation.

\section{Discussion}

With a rise in global obesity rates and parallel increasing bariatric surgeries performed, we are seeing more massive weight loss patients who are being referred to plastic surgeons for consideration for an abdominoplasty. In the United States alone, the number of abdominal plasties performed has been shown to increase 5-fold over a 12 year period at the turn of the century [8]. As increasing numbers of massive weight loss are presenting for consideration of abdominoplasty, it is vital to ensure the most appropriate surgical option is provided for best cosmetic outcome with minimal associated risks. Massive weight loss patients present with specific challenges such as supraumbilical skin excess which standard abdominoplasty may not address adequately. The FDL technique is ideal for the massive weight loss patient [5].

A literature review showed complication rates vary widely for the FDL series (12\%-62\%). Dellon's case series of 16 patients revealed an overall complication rate of 50\% [4]. Ramsey-Stewart's case series on 45 patients showed an overall complication rate of $13.3 \%$, describing partial wound healing and seromaas main complications in their study [7].

Duff et al. [8] described a larger and more recent retrospective case series review on $68 \mathrm{FDL}$ patients. The study noted an overall complication in 42 patients (62\%). Delayed wound healing was the most common complication $(n=17)$. In their study, 12 patients required revision operation for aesthetic complications. More importantly, they showed $82.4 \%$ of the patients achieved satisfactory cosmetic results in a single operation.

Finally, in the most recent and largest study of 100 post bariatric patients who undergone the FDL operation, the overall complication rate was 20\% [9]. 8 patients developed seroma, 9 patients had haematoma, 3 developed cellulitis, 1 had partial umbilical necrosis and 1 had wound dehiscence.

The results of our study were comparable to those previously published [10]. We had lower seroma rates and no haematoma postoperatively. We believe careful planning, meticulous techniques and a good understanding of fundamentals of surgical principles forms the basis of our successful experience with the FDL technique in our unit. However, we recognise there are limitations to our study in that it is 
Citation: Alethea T, Siva V, Sambasiva RG (2013) Fleur De Lys Abdominoplasty: Techniques to Reduce Complication Rates - Our Experience. Surgery Curr Res 3: 148. doi:10.4172/2161-1076.1000148

relatively small. It is carried out retrospectively and is therefore limited by the quality of documentation [11].

\section{Conclusion}

Our experience shows FDL is a safe approach in carefully selected patients with complications rates comparable to that previously published. The senior author's improvisation on current FDL technique has been described here, and has shown to reduce complication risks in a procedure that is viewed to be associated with significant complications. To conclude, we would like to share our 7 important technical tips to reduce complications:

1. Pinch and reduce by an inch on either side' of maximal width of vertical ellipse during pre-operative marking

2. Ligating and/or coagulating perforators before division, thereby preventing ends of perforators from retracting and subsequent potential bleeding points post-operatively

3. No undermining of flaps of the abdomen - facilitated by reducing the width to the vertical ellipse

4. Wash and second look for potential bleeding points before closure and appropriate haemostasis

5. No 'breaking of table' before closure of transverse incision

6. The placement of two drains - one under the vertical ellipse excision and one under the transverse abdomen excision

7. Post-operatively, ensure abdominal corset e.g. Tubipad is applied and encourage early mobilisation
The author and senior author have no conflict of interest to declare.

This study did not receive any funding source from either private or government sector.

\section{References}

1. Grazer FM (1973) Abdominoplasty. Plast Reconstr Surg 51: 617-623.

2. Castañares S, Goethel JA (1967) Abdominal lipectomy: a modification in technique. Plast Reconstr Surg 40: 378-383.

3. Dellon AL (1985) Fleur-de-lis abdominoplasty. Aesthetic Plast Surg 9: 27-32.

4. Friedman T, O'Brien Coon D, Michaels J, Purnell C, Hur S, et al. (2010) Fleurde-Lis abdominoplasty: a safe alternative to traditional abdominoplasty for the massive weight loss patient. Plast Reconstr Surg 125: 1525-1535.

5. WHO: Obesity and overweight. Fact sheet $\mathrm{N}^{\circ} 311$.

6. Ramsey-Stewart G (1993) Radical "Fleur-de-Lis" Abdominal after Bariatric Surgery. Obes Surg 3: 410-414.

7. Arthurs ZM, Cuadrado D, Sohn V, Wolcott K, Lesperance K, et al. (2007) Postbariatric panniculectomy: pre-panniculectomy body mass index impacts the complication profile. Am J Surg 193: 567-570.

8. Duff CG, Aslam S, Griffiths RW (2003) Fleur-de-Lys abdominoplasty--a consecutive case series. Br J Plast Surg 56: 557-566.

9. Borud LJ, Warren AG (2007) Modified vertical abdominoplasty in the massive weight loss patient. Plast Reconstr Surg 119: 1911-1921.

10. Overall Trends (2012) National Cosmetic Procedures. American Society of Plastic Surgeons.

11. O'Brien JA, Broderick GB, Hurwitz ZM, Montilla R, Castle J, et al. (2012) Fleurde-lis panniculectomy after bariatric surgery: our experience. Ann Plast Surg 68: $74-78$ 